\title{
Ab initio calculations with non-symmetrized hyperspherical har- monics for realistic NN potential models
}

\author{
Sergio Deflorian ${ }^{1,2, a}$, Nir Barnea ${ }^{3}$, Winfried Leidemann ${ }^{1,2}$, and Giuseppina Orlandini ${ }^{1,2}$ \\ ${ }^{1}$ Dipartimento di Fisica, Università di Trento, I-38123 Trento, Italy \\ ${ }^{2}$ Istituto Nazionale di Fisica Nucleare, Gruppo Collegato di Trento, I-38123 Trento, Italy \\ ${ }^{3}$ Racah Institute of Physics, The Hebrew University, 91904, Jerusalem, Israel
}

\begin{abstract}
This contribution deals with the problem of implementing the Pauli principle in a variational calculation of the binding energy of a fermionic system on the hyperspherical harmonics $(\mathrm{HH})$ basis. We summarize the main points of a method that avoids the antysimmetrization of the basis functions. In fact this gets increasingly cumbersome as the number of particles increases. The method is tested on the binding energies of two nuclei $\left({ }^{4} \mathrm{He}\right.$ and $\left.{ }^{6} \mathrm{Li}\right)$, using various nuclear potential models, including a realistic one.
\end{abstract}

\section{Introduction}

In order to antisymmetrize the hyperspherical harmonics (HH) basis functions an efficient symmetrization method had been developed in [1] and successfully applied in many applications up to a number of particle $\mathrm{N}=6$. However, this method requires considerable computational resources as the number of particles increases.

Recently a different strategy has been proposed [2, 3], where the HH symmetrization is avoided by using non-symmetrized HH (NSHH). The approach has been limited to treat central (also spin dependent) potentials. What we present here is a somewhat modified approach, which has allowed to use isospin dependent non central interactions and therefore also nuclear realistic potentials.

\section{Outline of the non-symmetrized $\mathrm{HH}$ approach}

In all traditional constructions of the $\mathrm{HH}$ functions in terms of the Jacobi coordinates (see e.g. [4]) they turn out not having well-defined permutational symmetries. If one wants to symmetrize them one has to deal with particle permutations which change the definition of the Jacobi coordinates. The couplings between the different angular momenta are changed as well, making the effect of particle permutation on the $\mathrm{HH}$ functions rather complicated. In particular the matrices that represent the permutation operators on the $\mathrm{HH}$ basis are full matrices which must be calculated numerically.

The idea of the NSHH approach in [2,3] consists of two steps. The first step aims at reducing the evaluation of the potential matrix to a one-dimensional integral. In particular one wants to reduce the matrix element of each potential term $V_{i j}$ on the (non-symmetrized) $\mathrm{HH}$ basis to an integral in the

\footnotetext{
ae-mail: s.deflorian.2@unitn.it
} 
pivot Jacobi coordinate $\vec{\eta}_{N-1}=\sqrt{\frac{1}{2}}\left(\vec{r}_{N-1}-\vec{r}_{N}\right)$, only. This requires that for each $(i, j)$ one applies a sequence of (adjacent) particle permutations $P_{i, i+1}$ that finally change particle $j$ into particle $N-1$ and particle $i$ into particle $N$. Due to the properties of the HHs, the matrices representing the permutation operator $P_{i, i+1}$ are block diagonal and can be easily calculated. There is a price to pay following this procedure. In fact, since the $\mathrm{HH}$ have no definite permutational symmetry, to each value of the hyperangular momentum $K$ correspond many more $\mathrm{HH}$ components than if one had already selected the symmetry. Therefore to a fixed $K_{\max }$ in the hyperangular momentum expansion correspond a basis of much larger size and therefore a larger Hamiltonian matrix. However, this is largely compensated by having to deal simply with the product of many large, but block diagonal matrices. One then proceeds to diagonalize the Hamiltonian to obtain eigenvalues and eigenstates. Since the Casimir operator of the permutation group $C(N)=\sum_{i, j}^{N} P_{i j}$ commutes with the Hamiltonian one is sure that the eigenvectors have well defined permutational symmetries.

The second step consists then in analyzing the symmetry of the eigenstates by applying the Casimir operator to each of them. Finally, the combination with spin (and isospin) wave functions of appropriate symmetry gives the totally antisymmetric wave function.

As already mentioned the procedure described here has been applied to systems interacting via central potentials (also spin dependent). In this case the total angular momentum $L$ and total spin $S$ are good quantum numbers. Accordingly, the basis can be identified by $(L, S) J^{\pi}$ (the parity $\pi$ corresponds to consider even or odd $K$ values in the expansion). Non-central potentials and in particular realistic nuclear potentials require to deal with basis functions in the J-coupling. Unfortunately this leads to an increase in the size of the Hamiltonian matrix and therefore in the number of eigenfunctions that need to be analyzed in terms of symmetry.

\subsection{How to avoid the symmetry analysis of the states}

We redefine the Hamiltonian adding a potential term proportional just to the Casimir operator:

$$
H^{\prime}=H+\gamma C(N)
$$

where $\gamma$ is a real parameter. Then one has

$$
E_{n, \Gamma}^{\prime}=E_{n, \Gamma}+\gamma \lambda_{\Gamma}
$$

where $E_{n, \Gamma}$ are the eigenvalues of $H$ with symmetry $\Gamma$ ( i.e. $\Gamma$ stands for symmetric (S), antisymmetric (A) or any mixed (M) symmetry) and $\lambda_{\Gamma}$ indicate the eigenvalues of the Casimir operator. We remind that the antisymmetric states correspond to the lowest eigenvalue of $C(N)$ i.e. $\lambda_{A}=-N(N-1) / 2$. This is ensured by choosing $\gamma$ larger than the lowest eigenstate of $H$, normalized to the number of particles [5]. Since we aim at obtaining the lowest antisymmetric eigenvalue of $H$ of a fermionic system, namely $E_{0, A}$, we will choose $\gamma>0$ and large enough so that $E_{0, A}^{\prime}$ is by far the lowest eigenvalue of $H^{\prime}$. The ground state energy is then obtained by

$$
E_{0, A}=E_{0, A}^{\prime}-\gamma \frac{N(N-1)}{2}
$$

The advantage of the procedure outlined here is in the possibility to obtain $E_{0, A}$ by the Lanczos algorithm, with a rather limited number of steps. 
INPC 2013

Table 1. Convergence rate in $K_{\max }$ of the ground state energy $E_{0, A}[\mathrm{MeV}]$ and $r_{r m s}[\mathrm{fm}]$. Results for ${ }^{4} \mathrm{He}$ are obtained with the AV4'(plus Coulomb) and AV18 potentials. Results for ${ }^{6} \mathrm{Li}$ are obtained with MTI-III.

\begin{tabular}{|c|c|c|c|c|c|c|}
\hline & ${ }^{4} \mathrm{He}$ & ${ }^{4} \mathrm{He}$ & ${ }^{4} \mathrm{He}$ & ${ }^{4} \mathrm{He}$ & ${ }^{6} \mathrm{Li}$ & \\
\hline & AV4' & AV4' & AV18 & AV18 & MTI-III & MTI-III \\
\hline$K_{\max }$ & $E_{0, A}$ & $r_{r m s}$ & $E_{0, A}$ & $r_{r m s}$ & $E_{0, A}$ & $r_{r m s}$ \\
\hline 2 & -32.258 & 1.3698 & -24.640 & 1.5063 & -46.99 & 2.161 \\
\hline 4 & -32.227 & 1.3840 & -26.124 & 1.5111 & -36.69 & 2.079 \\
\hline 6 & -31.781 & 1.3957 & -25.311 & 1.5061 & -36.45 & 2.120 \\
\hline 8 & -32.579 & 1.3798 & -24.999 & 1.5089 & -36.38 & 2.138 \\
\hline 10 & -31.858 & 1.3883 & -24.442 & 1.5197 & -36.32 & 2.156 \\
\hline 12 & -32.201 & 1.3866 & -24.491 & 1.5176 & -36.27 & 2.180 \\
\hline 14 & -32.047 & 1.3865 & -24.348 & 1.5184 & & \\
\hline 16 & -32.068 & 1.3865 & -24.313 & 1.5181 & & \\
\hline 18 & -32.051 & 1.3865 & -24.271 & 1.5177 & & \\
\hline 20 & -32.060 & 1.3865 & -24.266 & 1.5176 & & \\
\hline 22 & -32.049 & 1.3862 & -24.246 & 1.5170 & & \\
\hline 24 & -32.054 & 1.3863 & & & & \\
\hline GFMC[7] & $-32.11(2)$ & & & & & \\
\hline HН[8] & & & -24.22 & 1.512 & -35.91 & \\
\hline EIHH[6] & & & & & -36.6 & 2.15 \\
\hline FY[9] & & & -24.23 & & & \\
\hline FY[10] & & & -24.22 & 1.516 & & \\
\hline AGS[11] & & & -24.24 & & & \\
\hline
\end{tabular}

\section{Results}

The results presented in this section are obtained using various two-body nuclear interactions. The convergence in the $\mathrm{HH}$ basis is accelerated via a hyperradial dependent effective interaction derived by means of a unitary transformation (EIHH approach [6]). In table 1 the ground state energies $E_{0, A}$ and root mean square radii $\left(r_{r m s}\right)$ of ${ }^{4} \mathrm{He}$ and ${ }^{6} \mathrm{Li}$ are reported [5]. For ${ }^{4} \mathrm{He}$ we show results obtained with AV4' and AV18 [12] potentials, for ${ }^{6} \mathrm{Li}$ those obtained with MTI-III [13]. Table 1 shows the convergence rate and one can notice the good agreement with the results present in the literature, derived by other approaches. Our method is very efficient since the lowest eigenenergy of $H^{\prime}$ (and consequently the ground state energy of $H$ ) can be found with a rather limited number of steps of the Lanczos algorithm. This opens up the possibility to reach accurate $\mathrm{HH}$ results also for larger nuclear systems.

\section{References}

[1] N. Barnea and A. Novoselsky, Phys. Rev. A 57, 48 (1998); Ann. Phys. (N.Y.) 256, 192 (1997)

[2] M. Gattobigio, A. Kievsky, M. Viviani, and P. Barletta, Phys. Rev. A 79, 032513 (2009); FewBody Syst. 45, 127 (2009).

[3] M. Gattobigio, A. Kievsky, and M. Viviani, Phys. Rev. C 83, 024001 (2011).

[4] V. D. Efros, Yad. Fiz. 15, 226 (1972) [Sov. J. Nucl. Phys. 15, 128 (1972)] 
[5] S. Deflorian, N. Barnea, W.Leidemann, and G. Orlandini, Few Body Syst. in print, published on line DOI 10.1007/S00601-013-0717-y

[6] N. Barnea, W. Leidemann, and G. Orlandini, Phys. Rev. C 61, 54001 (2000); Nucl. Phys. A 693, 565 (2001)

[7] R.B. Wiringa and S.C. Pieper, Phys. Rev. Lett. 89, 182501 (2002)

[8] M. Viviani, A. Kievsky and S. Rosati, Phys. Rev. C 71, 24006 (2005); A. Kievsky, S. Rosati, M. Viviani, L.E. Marcucci and L. Girlanda, Journal of Physics G: Nuclear and Particle Physics, 35, 63101 (2008)

[9] A. Nogga, H. Kamada, W. Glöckle, and B.R. Barrett, Phys. Rev. C 65, 054003 (2002)

[10] R. Lazauskas and J. Carbonell, Phys. Rev. C 70, 044002 (2004)

[11] A. Deltuva and A.C. Fonseca, Phys. Rev. C 75, 014005 (2007)

[12] R.B. Wiringa, V.G.J. Stoks, and R. Schiavilla, Phys. Rev. C 51, 38 (1995); R.B. Wiringa and S.C. Pieper, Phys. Rev. Lett. 89, 182501 (2002)

[13] R.A. Malfliet and A. Tjon, Nucl. Phys. A 127, 161 (1969) 